\title{
ON THE CLASSIFICATION BY MORIMOTO AND NAGANO
}

\author{
ALEXANDER ISAEV
}

\begin{abstract}
We consider a family $M_{t}^{3}$, with $t>1$, of real hypersurfaces in a complex affine 3-dimensional quadric arising in connection with the classification of homogeneous compact simply-connected real-analytic hypersurfaces in $\mathbb{C}^{n}$ due to Morimoto and Nagano. To finalize their classification, one needs to resolve the problem of the CR-embeddability of $M_{t}^{3}$ in $\mathbb{C}^{3}$. In our earlier article we showed that $M_{t}^{3}$ is CR-embeddable in $\mathbb{C}^{3}$ for all $1<t<\sqrt{(2+\sqrt{2}) / 3}$. In the present paper we prove that $M_{t}^{3}$ can be immersed in $\mathbb{C}^{3}$ for every $t>1$ by means of a polynomial map. In addition, one of the immersions that we construct helps simplify the proof of the above CR-embeddability theorem and extend it to the larger parameter range $1<t<\sqrt{5} / 2$.
\end{abstract}

\section{INTRODUCTION}

This paper concerns the following classical problem investigated by Morimoto and Nagano in [MN]: determine all compact simply-connected real-analytic hypersurfaces in $\mathbb{C}^{n}$ homogeneous under an action of a Lie group by CR-transformations. It was shown in [MN] that every such hypersurface is CR-equivalent to either the sphere $S^{2 n-1}$ or, for $n=3,7$, to a manifold from the 1-parameter family $M_{t}^{n}$ as defined below.

To introduce $M_{t}^{n}$ for any $n \geqslant 2$, consider the $n$-dimensional affine quadric in $\mathbb{C}^{n+1}$ :

$$
Q^{n}:=\left\{\left(z_{1}, \ldots, z_{n+1}\right) \in \mathbb{C}^{n+1}: z_{1}^{2}+\cdots+z_{n+1}^{2}=1\right\} .
$$

The group $\mathrm{SO}(n+1, \mathbb{R})$ acts on $Q^{n}$, with the orbits of the action being the sphere $S^{n}=Q^{n} \cap \mathbb{R}^{n+1}$ as well as the compact strongly pseudoconvex hypersurfaces

$$
M_{t}^{n}:=\left\{\left(z_{1}, \ldots, z_{n+1}\right) \in \mathbb{C}^{n+1}:\left|z_{1}\right|^{2}+\cdots+\left|z_{n+1}\right|^{2}=t\right\} \cap Q^{n}, t>1,
$$

which are simply-connected for $n \geqslant 3$. These hypersurfaces are all nonspherical (see [I1, Remark 2.2]) and pairwise CR-nonequivalent (see [KZ, Example 13.9], $\left[\mathrm{BH}\right.$, Theorem 2]). They are the boundaries of Grauert tubes around $S^{n}$ (note that $Q^{n}$ can be naturally identified with the tangent bundle $T\left(S^{n}\right)$ ). In [MN], Morimoto and Nagano did not investigate the question of whether or not $M_{t}^{n}$ admits a realanalytic CR-embedding in $\mathbb{C}^{n}$ for $n=3,7$, thus their classification in these two dimensions was not finalized.

The family $M_{t}^{n}$ was studied in our papers [I1], [I2]. In particular, in [I1, Corollary $2.1]$ we observed that a necessary condition for the existence of a real-analytic CRembedding of $M_{t}^{n}$ in $\mathbb{C}^{n}$ is the embeddability of the sphere $S^{n}$ in $\mathbb{C}^{n}$ as a totally real submanifold. The problem of the existence of a totally real embedding of $S^{n}$ in $\mathbb{C}^{n}$ was considered by Gromov (see [G1] and [G2, p. 193]), Stout-Zame (see [SZ]), Ahern-Rudin (see [AR]), Forstnerič (see [F1], [F2], [F3]). In particular, $S^{n}$ was shown to admit a smooth totally real embedding in $\mathbb{C}^{n}$ only for $n=3$, hence $M_{t}^{7}$ cannot be real-analytically CR-embedded in $\mathbb{C}^{7}$. On the other hand, since $S^{3}$ is a totally real submanifold of $Q^{3}$, any real-analytic totally real embedding of $S^{3}$ in $\mathbb{C}^{3}$

Mathematics Subject Classification: 32C09, 32V30.

Keywords: immersions and embeddings of CR-manifolds in complex space. 
(which is known to exist, for instance, by [AR]) extends to a biholomorphic map defined in a neighborhood of $S^{3}$ in $Q^{3}$. Owing to the fact that $M_{t}^{3}$ accumulate to $S^{3}$ as $t \rightarrow 1$, this observation implies that $M_{t}^{3}$ admits a real-analytic CR-embedding in $\mathbb{C}^{3}$ for all $t$ sufficiently close to 1 . Thus, the classification of homogeneous compact simply-connected real-analytic hypersurfaces in complex dimension 3 is special as it contains manifolds other than the sphere $S^{5}$.

More precisely, in [I2, Theorem 1.1] we showed that $M_{t}^{3}$ embeds in $\mathbb{C}^{3}$ for all $1<t<\sqrt{(2+\sqrt{2}) / 3}$ by means of a real-analytic CR-map. This was proved by analyzing the holomorphic continuation of the explicit polynomial totally real embedding of $S^{3}$ in $\mathbb{C}^{3}$ constructed in [AR] (see Remark 4.3 for details), and the argument was quite involved computationally. Since the hypersurfaces in the family $M_{t}^{3}$ are all pairwise CR-nonequivalent, [I2, Theorem 1.1] leaves the problem of the CR-embeddability of $M_{t}^{3}$ in $\mathbb{C}^{3}$ for $t \geqslant \sqrt{(2+\sqrt{2}) / 3}$ completely open. In this paper, we make steps towards resolving this problem and also look at related matters.

Even the question of whether every $M_{t}^{3}$ can be immersed in $\mathbb{C}^{3}$ is nontrivial. The answer to this question is positive as the quadric $Q^{n}$ is known to admit a holomorphic immersion in $\mathbb{C}^{n}$ for every $n \geqslant 2$ (see [BN, p. 19]). However, such an immersion cannot be algebraic. In our first main result we show that, nevertheless, an algebraic immersion exists for each $M_{t}^{3}$ :

THEOREM 1.1. Every hypersurface $M_{t}^{3}$ with $t>1$ can be immersed in $\mathbb{C}^{3}$ by means of a polynomial map $\mathbb{C}^{4} \rightarrow \mathbb{C}^{3}$.

In the proof of Theorem 1.1 in the next section, for every $n \in \mathbb{N}$ we explicitly construct a polynomial map $F_{n}: \mathbb{C}^{4} \rightarrow \mathbb{C}^{3}$ that yields an immersion of $M_{t}^{3}$ for all $1<t<t_{n}$, where $t_{n} \rightarrow \infty$ as $n \rightarrow \infty$. None of the maps $F_{n}$ is injective on $M_{t}^{3}$ if $t \geqslant \sqrt{2}$, thus these maps certainly cannot be used to establish the CR-embeddability of $M_{t}^{3}$ in $\mathbb{C}^{3}$ for $t \geqslant \sqrt{2}$ (see Remark 4.1). We did not investigate $F_{n}$ for injectivity on $M_{t}^{3}$ if $\sqrt{(2+\sqrt{2}) / 3} \leqslant t<\sqrt{2}$ and $n>1$. It is possible that for some $t$ in this range and sufficiently large $n$ the immersions of $M_{t}^{3}$ given by the maps $F_{n}$ are in fact embeddings, but the calculations required to verify the injectivity of $F_{n}$ on $M_{t}^{3}$ for $n>1$ appear to be rather prohibiting. In fact, even studying the fibers of a much simpler polynomial map considered in [I2] was computationally quite hard.

At the same time, the first map $F_{1}$ in the sequence $\left\{F_{n}\right\}$ turns out to be easier to handle than its counterpart studied in [I2], which allows us to improve [I2, Theorem 1.1] and obtain our second main result:

THEOREM 1.2. The hypersurface $M_{t}^{3}$ admits a real-analytic $C R$-embeddings in $\mathbb{C}^{3}$ if $1<t<\sqrt{5} / 2$.

Note that the bound $\sqrt{5} / 2$ that appears in Theorem 1.2 is only slightly greater than the bound $\sqrt{(2+\sqrt{2}) / 3}$ of [I2, Theorem 1.1]. Thus, our purpose here was not so much to enlarge the range of $t$ as to produce a more transparent argument for CR-embeddability, which we were able to achieve by invoking the map $F_{1}$ instead of the map arising from [AR]. Theorem 1.2 is established in Section 3.

Our construction of the sequence $\left\{F_{n}\right\}$ is to some extent inspired by article [AR], in which harmonic polynomials were used to produce the map presented in the main theorem therein (see Remark 4.3). However, we do not explicitly utilize harmonicity; rather, we directly come up with suitable polynomial immersions. The maps $F_{n}$ are also of independent interest as each of them yields an explicit totally real embedding of $S^{3}$ in $\mathbb{C}^{3}$. It then follows that each $F_{n}$ defines a CR-embedding of $M_{t}^{3}$ to $\mathbb{C}^{3}$ for $1<t<\tau_{n}$, where $\tau_{n} \leqslant \sqrt{2}$ (see Remark 4.2). 
Acknowledgment. We thank László Lempert for drawing our attention to reference $[\mathrm{BH}]$ and Stefan Nemirovski for many useful discussions. This work was done while the author was visiting the Steklov Mathematical Institute in Moscow. The research is supported by the ARC Discovery Grant DP190100354.

\section{Proof of Theorem 1.1}

For convenience, we will argue in the coordinates

$$
w_{1}:=z_{1}+i z_{2}, w_{2}:=z_{1}-i z_{2}, w_{3}:=z_{3}+i z_{4}, w_{4}:=z_{3}-i z_{4} .
$$

In these coordinates the quadric $Q^{3}$ becomes

$$
\left\{\left(w_{1}, w_{2}, w_{3}, w_{4}\right) \in \mathbb{C}^{4}: w_{1} w_{2}+w_{3} w_{4}=1\right\}
$$

(see (1.1)), the sphere $S^{3} \subset Q^{3}$ becomes

$$
\left\{\left(w_{1}, w_{2}, w_{3}, w_{4}\right) \in \mathbb{C}^{4}: w_{2}=\bar{w}_{1}, w_{4}=\bar{w}_{3}\right\} \cap Q^{3},
$$

and the hypersurface $M_{t}^{3} \subset Q^{3}$ becomes

$$
\left\{\left(w_{1}, w_{2}, w_{3}, w_{4}\right) \in \mathbb{C}^{4}:\left|w_{1}\right|^{2}+\left|w_{2}\right|^{2}+\left|w_{3}\right|^{2}+\left|w_{4}\right|^{2}=2 t\right\} \cap Q^{3}
$$

$($ see $(1.2))$.

Let $F: \mathbb{C}^{4} \rightarrow \mathbb{C}^{3}$ be a map of the form

$$
\left(w_{1}, w_{2}, w_{3}, w_{4}\right) \mapsto\left(w_{1}, w_{3}, f\left(w_{1}, w_{2}, w_{3}, w_{4}\right)\right),
$$

where $f$ is an entire function. Clearly, $F$ yields an immersion of $M_{t}^{3}$ in $\mathbb{C}^{3}$ if and only if its restriction $\tilde{F}:=\left.F\right|_{Q^{3}}$ is nondegenerate at every point of $M_{t}^{3}$. We need the following fact.

Lemma 2.1. The map $\tilde{F}$ is nondegenerate at a point $w^{0}=\left(w_{1}^{0}, w_{2}^{0}, w_{3}^{0}, w_{4}^{0}\right) \in Q^{3}$ if and only if one has

$$
w_{3}^{0} \frac{\partial f}{\partial w_{2}}\left(w^{0}\right)-w_{1}^{0} \frac{\partial f}{\partial w_{4}}\left(w^{0}\right) \neq 0
$$

Proof. Observe that $\left|w_{1}\right|+\left|w_{3}\right|>0$ on $Q^{3}$ (see (2.1)). For $w_{1} \neq 0$ we choose $w_{1}, w_{3}, w_{4}$ as local coordinates on $Q^{3}$ and write the third component of $\tilde{F}$ as

$$
\varphi:=f\left(w_{1}, \frac{1-w_{3} w_{4}}{w_{1}}, w_{3}, w_{4}\right)
$$

In this coordinate chart the Jacobian $J_{\tilde{F}}$ of $\tilde{F}$ is calculated as

$$
J_{\tilde{F}}=\frac{\partial \varphi}{\partial w_{4}}=-\frac{w_{3}}{w_{1}} \frac{\partial f}{\partial w_{2}}+\frac{\partial f}{\partial w_{4}}
$$

hence it is nonvanishing at $w^{0} \in Q^{3}$ with $w_{1}^{0} \neq 0$ if and only if (2.5) holds.

Analogously, if $w_{3} \neq 0$, we choose $w_{1}, w_{2}, w_{3}$ as local coordinates on $Q^{3}$ and write the third component of $\tilde{F}$ as

$$
\psi:=f\left(w_{1}, w_{2}, w_{3}, \frac{1-w_{1} w_{2}}{w_{3}}\right) .
$$

In this coordinate chart we have

$$
J_{\tilde{F}}=-\frac{\partial \psi}{\partial w_{2}}=-\frac{\partial f}{\partial w_{2}}+\frac{w_{1}}{w_{3}} \frac{\partial f}{\partial w_{4}},
$$

hence $J_{\tilde{F}}$ does not vanish at $w^{0} \in Q^{3}$ with $w_{3}^{0} \neq 0$ if and only if condition (2.5) is satisfied. 
We will now construct a sequence $\left\{F_{n}\right\}_{n \geqslant 1}$ of maps of the form (2.4):

$$
F_{n}\left(w_{1}, w_{2}, w_{3}, w_{4}\right)=\left(w_{1}, w_{3}, P_{n}\left(w_{1}, w_{2}, w_{3}, w_{4}\right)\right),
$$

where $P_{n}$ is a polynomial, having the property: there exists $t_{n}>1$ such that

$$
w_{3} \frac{\partial P_{n}}{\partial w_{2}}-w_{1} \frac{\partial P_{n}}{\partial w_{4}} \neq 0 \text { on } M_{t}^{3} \text { for } 1<t<t_{n}
$$

and

$$
t_{n} \rightarrow \infty \text { as } n \rightarrow \infty .
$$

Lemma 2.1 will then imply the theorem.

Our strategy for coming up with polynomials $P_{n}$ as above is as follows:

(A) Choose some polynomials $R_{n}$ having the property: there exists $t_{n}>1$ such that

$$
R_{n} \neq 0 \text { on } M_{t}^{3} \text { for } 1<t<t_{n},
$$

and (2.8) holds.

(B) For each $n$ find a polynomial $P_{n}$ that solves the equation

$$
w_{3} \frac{\partial P_{n}}{\partial w_{2}}-w_{1} \frac{\partial P_{n}}{\partial w_{4}}=R_{n} \text { everywhere on } \mathbb{C}^{4} \text {. }
$$

By (2.9), the polynomials $P_{n}$ will satisfy (2.7) as required.

Coming up with suitable polynomials $R_{n}$ in Part (A) such that there are solutions to (2.10) in Part (B) is not easy. After much computational experimentation, we discovered that polynomials of the following form work well:

$$
R_{n}:=\left(\left(\frac{1}{2}+i a_{n}\right) w_{1} w_{2}-\left(\frac{1}{2}-i a_{n}\right) w_{3} w_{4}\right)^{2 n},
$$

where $a_{n}$ are positive numbers to be chosen later.

Let us study the zeroes of $R_{n}$ on $M_{t}^{3}$. First of all, we restrict $R_{n}$ to $Q^{3}$ by replacing $w_{1} w_{2}$ with $1-w_{3} w_{4}$ :

$$
\left.R_{n}\right|_{Q^{3}}=\left(w_{3} w_{4}-\left(\frac{1}{2}+i a_{n}\right)\right)^{2 n}
$$

(see $(2.1))$. Thus, $R_{n}$ vanishes at a point $w=\left(w_{1}, w_{2}, w_{3}, w_{4}\right) \in Q^{3}$ if and only if

$$
w_{1} w_{2}=\frac{1}{2}-i a_{n}, \quad w_{3} w_{4}=\frac{1}{2}+i a_{n} .
$$

Such a point lies in $M_{t}^{3}$ for some $t>1$ if and only if

$$
\left|w_{1}\right|^{2}+\left|\frac{1}{2}-i a_{n}\right|^{2} \frac{1}{\left|w_{1}\right|^{2}}+\left|w_{3}\right|^{2}+\left|\frac{1}{2}+i a_{n}\right|^{2} \frac{1}{\left|w_{3}\right|^{2}}=2 t
$$

(see (2.3)), or, equivalently, if and only if

$$
\left|w_{1}\right|^{2}+\left(\frac{1}{4}+a_{n}^{2}\right) \frac{1}{\left|w_{1}\right|^{2}}+\left|w_{3}\right|^{2}+\left(\frac{1}{4}+a_{n}^{2}\right) \frac{1}{\left|w_{3}\right|^{2}}=2 t .
$$

The following elementary lemma, which we state without proof, will often be helpful:

Lemma 2.2. For fixed $p>0$, let

$$
g(x):=x+\frac{p}{x}, \quad x>0 .
$$

Then $\min _{x>0} g(x)=2 \sqrt{p}$. 
Letting in Lemma 2.2

$$
p=\frac{1}{4}+a_{n}^{2}
$$

from (2.13) we see

$$
t \geqslant 2 \sqrt{\frac{1}{4}+a_{n}^{2}}
$$

We then set

$$
t_{n}:=2 \sqrt{\frac{1}{4}+a_{n}^{2}}
$$

Clearly, with this choice of $t_{n}$ condition (2.9) holds. To satisfy (2.8), the positive numbers $a_{n}$ will be chosen to increase to $\infty$.

Remark 2.3. Notice that the map $\tilde{F}_{n}:=\left.F_{n}\right|_{Q^{3}}$ degenerates at some point of $M_{t}^{3}$ whenever $t \geqslant t_{n}$. Indeed, for any such $t$ find $x_{0}>0$ satisfying $g\left(x_{0}\right)=t$. Then the point

$$
\left(\sqrt{x_{0}}, \frac{\frac{1}{2}-i a_{n}}{\sqrt{x_{0}}}, \sqrt{x_{0}}, \frac{\frac{1}{2}+i a_{n}}{\sqrt{x_{0}}}\right)
$$

lies in $M_{t}^{3}$, and $\tilde{F}_{n}$ is degenerate at it.

Let us now turn to Part (B). We fix $n \geqslant 1$ and look for a solution to equation (2.10) in the form

$$
P_{n}=\sum_{k=1}^{2 n} \alpha_{k} w_{1}^{2 n-k} w_{2}^{2 n-k+1} w_{3}^{k-1} w_{4}^{k},
$$

where $\alpha_{j}$ are complex numbers, which will be computed in terms of $a_{n}$ shortly. With $P_{n}$ given by $(2.15)$, the left-hand side of $(2.10)$ is

$$
\begin{aligned}
& -\alpha_{1}\left(w_{1} w_{2}\right)^{2 n}+\alpha_{2 n}\left(w_{3} w_{4}\right)^{2 n}+ \\
& \sum_{k=1}^{2 n-1}\left((k+1) \alpha_{2 n-k}-(2 n-k+1) \alpha_{2 n-k+1}\right)\left(w_{1} w_{2}\right)^{k}\left(w_{3} w_{4}\right)^{2 n-k} .
\end{aligned}
$$

Write the right-hand side of (2.10) as

$$
\begin{aligned}
& \left(\frac{1}{2}+i a_{n}\right)^{2 n}\left(w_{1} w_{2}\right)^{2 n}+\left(\frac{1}{2}-i a_{n}\right)^{2 n}\left(w_{3} w_{4}\right)^{2 n}+ \\
& \quad \sum_{k=1}^{2 n-1}(-1)^{k}\left(\begin{array}{c}
2 n \\
k
\end{array}\right)\left(\frac{1}{2}+i a_{n}\right)^{k}\left(\frac{1}{2}-i a_{n}\right)^{2 n-k}\left(w_{1} w_{2}\right)^{k}\left(w_{3} w_{4}\right)^{2 n-k} .
\end{aligned}
$$

Comparing (2.16) and (2.17), we, first of all, see

$$
\alpha_{1}=-\left(\frac{1}{2}+i a_{n}\right)^{2 n}, \quad \alpha_{2 n}=\left(\frac{1}{2}-i a_{n}\right)^{2 n} .
$$

Next, using the expression for $\alpha_{2 n}$ from (2.18), comparison of (2.16) and (2.17) for $k$ increasing from 1 to $n-1$ yields by induction

$$
\alpha_{2 n-k}=\frac{1}{k+1}\left(\begin{array}{c}
2 n \\
k
\end{array}\right) \sum_{\ell=0}^{k}(-1)^{\ell}\left(\frac{1}{2}+i a_{n}\right)^{\ell}\left(\frac{1}{2}-i a_{n}\right)^{2 n-\ell}, k=1, \ldots, n-1,
$$

in particular

$$
\alpha_{n+1}=\frac{1}{n}\left(\begin{array}{c}
2 n \\
n-1
\end{array}\right) \sum_{\ell=0}^{n-1}(-1)^{\ell}\left(\frac{1}{2}+i a_{n}\right)^{\ell}\left(\frac{1}{2}-i a_{n}\right)^{2 n-\ell} .
$$


Similarly, using the expression for $\alpha_{1}$ from (2.18), comparison of (2.16) and (2.17) for $k$ decreasing from $2 n-1$ to $n+1$ yields by induction

$$
\begin{aligned}
\alpha_{2 n-k+1}=-\frac{1}{2 n-k+1}\left(\begin{array}{c}
2 n \\
k
\end{array}\right) \sum_{\ell=0}^{2 n-k}(-1)^{\ell}\left(\frac{1}{2}+i a_{n}\right)^{2 n-\ell}\left(\frac{1}{2}-i a_{n}\right)^{\ell}, & \\
k & =n+1, \ldots, 2 n-1,
\end{aligned}
$$

in particular

$$
\alpha_{n}=-\frac{1}{n}\left(\begin{array}{c}
2 n \\
n+1
\end{array}\right) \sum_{\ell=0}^{n-1}(-1)^{\ell}\left(\frac{1}{2}+i a_{n}\right)^{2 n-\ell}\left(\frac{1}{2}-i a_{n}\right)^{\ell} .
$$

We will now compare the remaining terms of (2.16) and (2.17), namely, the ones corresponding to $k=n$ :

$$
(n+1)\left(\alpha_{n}-\alpha_{n+1}\right)=(-1)^{n}\left(\begin{array}{c}
2 n \\
n
\end{array}\right)\left(\frac{1}{2}+i a_{n}\right)^{n}\left(\frac{1}{2}-i a_{n}\right)^{n} .
$$

Invoking formulas $(2.19),(2.20)$ then leads to the following condition on $a_{n}$ :

$$
\sum_{\ell=0}^{2 n}(-1)^{\ell}\left(\frac{1}{2}+i a_{n}\right)^{\ell}\left(\frac{1}{2}-i a_{n}\right)^{2 n-\ell}=0 .
$$

Dividing by $\left(\frac{1}{2}-i a_{n}\right)^{2 n}$ and setting

$$
A_{n}:=-\frac{\frac{1}{2}+i a_{n}}{\frac{1}{2}-i a_{n}}
$$

we see that $(2.21)$ is equivalent to

$$
\sum_{\ell=0}^{2 n} A_{n}^{\ell}=0
$$

Summing up the first $2 n+1$ terms of the geometric series with common ratio $A$, we then obtain

$$
1-A^{2 n+1}=0
$$

or, equivalently,

$$
\operatorname{Re}\left(\frac{1}{2}+i a_{n}\right)^{2 n+1}=0 .
$$

Thus, $a_{n}$ must have the property that the value of the function $z^{2 n+1}$ at $\frac{1}{2}+i a_{n}$ is an imaginary number. Certainly, $z^{2 n+1}$ takes imaginary values on the line $\operatorname{Re} z=1 / 2$, and we let $\frac{1}{2}+i a_{n}$ be the point with the largest possible argument at which $\arg z^{2 n+1}=\pi / 2$. Namely, we choose $a_{n}>0$ so that

$$
\arg \left(\frac{1}{2}+i a_{n}\right)=\frac{\frac{\pi}{2}+2 \pi K}{2 n+1},
$$

where $K$ is the largest integer less than $n / 2$. It is then clear that (2.22) holds and

$$
\arg \left(\frac{1}{2}+i a_{n}\right) \rightarrow \frac{\pi}{2} \text { as } n \rightarrow \infty,
$$

hence the sequence $\left\{a_{n}\right\}$ converges to $\infty$. Therefore, by (2.14), condition (2.8) is satisfied.

We have thus found two sequences of polynomials $\left\{R_{n}\right\}$ and $\left\{P_{n}\right\}$ as required in Parts (A) and (B). The proof of the theorem is complete.

To give a better idea of the above argument, we will now write out details for $n=1$. This special case will be required for our proof of Theorem 1.2 in the next section. 
Example 2.4. Let $n=1$. Condition (2.23) yields

$$
a_{1}=\frac{1}{2 \sqrt{3}} \text {. }
$$

Hence, by (2.11) we have

$$
R_{1}=\left(\frac{1}{6}+\frac{i}{2 \sqrt{3}}\right)\left(w_{1} w_{2}\right)^{2}-\frac{2}{3} w_{1} w_{2} w_{3} w_{4}+\left(\frac{1}{6}-\frac{i}{2 \sqrt{3}}\right)\left(w_{3} w_{4}\right)^{2},
$$

and $(2.15),(2.18)$ lead to a formula for $P_{1}$ :

$$
P_{1}=-\left(\frac{1}{6}+\frac{i}{2 \sqrt{3}}\right) w_{1} w_{2}^{2} w_{4}+\left(\frac{1}{6}-\frac{i}{2 \sqrt{3}}\right) w_{2} w_{3} w_{4}^{2} .
$$

Further, by (2.14), we have

$$
t_{1}=\frac{2}{\sqrt{3}}
$$

Then, by Remark 2.3 , the map $\tilde{F}_{1}$ is nondegenerate at every point of $M_{t}^{3}$ if and only if $1<t<2 / \sqrt{3}$. We will investigate $\tilde{F}_{1}$ for injectivity in the next section.

\section{Proof of Theorem 1.2}

In order to produce a CR-embedding of $M_{t}^{3}$ in $\mathbb{C}^{3}$ for $1<t<\sqrt{5} / 2$ we will utilize the map $\tilde{F}_{1}$. It is clear from $(2.25)$ that $\sqrt{5} / 2<t_{1}$, thus we only need to show that $\tilde{F}_{1}$ is injective on each $M_{t}^{3}$ for $t$ in this range.

We start by studying the fibers of $\tilde{F}_{1}$.

Proposition 3.1. Let two points $w=\left(w_{1}, w_{2}, w_{3}, w_{4}\right)$ and $\hat{w}=\left(\hat{w}_{1}, \hat{w}_{2}, \hat{w}_{3}, \hat{w}_{4}\right)$ lie in $Q^{3}$ and assume that $\tilde{F}_{1}(w)=\tilde{F}_{1}(\hat{w})$. Then we have:

(a) $\hat{w}_{1}=w_{1}, \hat{w}_{3}=w_{3}$;

(b) if $w_{1}=0$ or $w_{3}=0$, then $\hat{w}=w$;

(c) if $w_{1} \neq 0$ and $w_{3} \neq 0$, then either $\hat{w}=w$ or one of the following holds:

$$
\begin{aligned}
& \hat{w}_{4}=-\frac{1+i \sqrt{3}}{2 w_{3}}\left(w_{3} w_{4}-1\right), \\
& \hat{w}_{4}=-\frac{1-i \sqrt{3}}{2 w_{3}}\left(w_{3} w_{4}-\frac{1+i \sqrt{3}}{2}\right) ;
\end{aligned}
$$

(d) neither of the two values in the right-hand side of (3.1) is equal to $w_{4}$ if $w \in M_{t}^{3}$ for $t<t_{1}$

(e) the two values in the right-hand side of (3.1) are distinct if $w \in M_{t}^{3}$ for $t<t_{1}$.

Hence, the fiber $\tilde{F}_{1}^{-1}\left(\tilde{F}_{1}(w)\right)$ consists of at most three points, and, if $w \in M_{t}^{3}$ with $w_{1} \neq 0, w_{3} \neq 0$ for $t<t_{1}$, it consists of exactly three points.

Proof. Part (a) is immediate from (2.6). Furthermore, (2.24) yields

$$
\begin{aligned}
-\left(\frac{1}{6}+\frac{i}{2 \sqrt{3}}\right) w_{1} \hat{w}_{2}^{2} \hat{w}_{4}+ & \left(\frac{1}{6}-\frac{i}{2 \sqrt{3}}\right) \hat{w}_{2} w_{3} \hat{w}_{4}^{2}= \\
& -\left(\frac{1}{6}+\frac{i}{2 \sqrt{3}}\right) w_{1} w_{2}^{2} w_{4}+\left(\frac{1}{6}-\frac{i}{2 \sqrt{3}}\right) w_{2} w_{3} w_{4}^{2},
\end{aligned}
$$

which together with (2.1) implies part (b).

From now on, we assume that $w_{1} \neq 0$ and $w_{3} \neq 0$. Then using (2.1) we substitute

$$
w_{2}=\frac{1-w_{3} w_{4}}{w_{1}}, \quad \hat{w}_{2}=\frac{1-w_{3} \hat{w}_{4}}{w_{1}}
$$


into (3.2) and simplifying the resulting expression obtain

$$
\begin{aligned}
\left(\hat{w}_{4}-w_{4}\right)\left[-\frac{w_{3}^{2}}{3} \hat{w}_{4}^{2}\right. & +\left(\left(\frac{1}{2}+\frac{i}{2 \sqrt{3}}\right) w_{3}-\frac{w_{3}^{2} w_{4}}{3}\right) \hat{w}_{4}+ \\
& \left.\left(\frac{1}{2}+\frac{i}{2 \sqrt{3}}\right) w_{3} w_{4}-\frac{w_{3}^{2} w_{4}^{2}}{3}-\left(\frac{1}{6}+\frac{i}{2 \sqrt{3}}\right)\right]=0 .
\end{aligned}
$$

We treat identity (3.4) as an equation with respect to $\hat{w}_{4}$. By part (a) and formula (3.3), the solution $\hat{w}_{4}=w_{4}$ leads to the point $w$. Further, the solutions of the quadratic equation given by setting the expression in the square brackets in (3.4) to zero, are shown in formula (3.1). This establishes part (c).

Next, for $\hat{w}_{4}=w_{4}$ the expression in the square brackets in (3.4) becomes

$$
-\left(w_{3} w_{4}-\left(\frac{1}{2}+\frac{i}{2 \sqrt{3}}\right)\right)^{2}=-\left.R_{1}\right|_{Q^{3}}
$$

(cf. $(2.12)$ ), which implies that $\tilde{F}_{1}$ is degenerate at $w$. This contradicts our choice of $w$ and thus establishes part (d).

Finally, the two values in (3.1) are easily seen to be equal if and only if

$$
w_{3} w_{4}=\frac{1}{2}+\frac{i}{2 \sqrt{3}}
$$

which leads to the same contradiction as in part (d), so part (e) follows. The proof is complete.

Proposition 3.1 implies that in order to establish Theorem 1.2 it suffices to show that for every value $1<t<\sqrt{5} / 2$ and every point

$$
w=\left(w_{1}, \frac{1-w_{3} w_{4}}{w_{1}}, w_{3}, w_{4}\right) \in M_{t}^{3} \text { with } w_{1} \neq 0, w_{3} \neq 0,
$$

the point $\hat{w}:=\left(w_{1},\left(1-w_{3} \hat{w}_{4}\right) / w_{1}, w_{3}, \hat{w}_{4}\right)$ does not lie in $M_{t}^{3}$ for any of the two choices of $\hat{w}_{4}$ in (3.1). In fact, we only need to consider the first solution in (3.1) as the second solution turns into the first one upon interchanging $w_{4}$ and $\hat{w}_{4}$.

Let now $\hat{w}$ correspond to the first choice of $\hat{w}_{4}$ in (3.1) and assume that $\hat{w} \in M_{t}^{3}$. Set $b:=w_{3} w_{4}, \hat{b}:=w_{3} \hat{w}_{4}$. Then by (3.1) we see

$$
\hat{b}=-\frac{1+i \sqrt{3}}{2}(b-1)
$$

We have

$$
\begin{aligned}
& \left|w_{1}\right|^{2}+\frac{|b-1|^{2}}{\left|w_{1}\right|^{2}}+\left|w_{3}\right|^{2}+\frac{|b|^{2}}{\left|w_{3}\right|^{2}}=2 t, \\
& \left|w_{1}\right|^{2}+\frac{|\hat{b}-1|^{2}}{\left|w_{1}\right|^{2}}+\left|w_{3}\right|^{2}+\frac{|\hat{b}|^{2}}{\left|w_{3}\right|^{2}}=2 t,
\end{aligned}
$$

where by (3.5) the second equation can be rewritten as

$$
\left|w_{1}\right|^{2}+\left|b-\frac{1+i \sqrt{3}}{2}\right|^{2} \frac{1}{\left|w_{1}\right|^{2}}+\left|w_{3}\right|^{2}+\frac{|b-1|^{2}}{\left|w_{3}\right|^{2}}=2 t
$$

By Lemma 2.2 it then follows that the point $b$ lies in the intersection of the interiors of two ellipses:

where

$$
\mathcal{D}:=\mathcal{E}_{1} \cap \mathcal{E}_{2}
$$

$$
\mathcal{E}_{1}:=\left\{z \in \mathbb{C}:|z-1|+|z|<\frac{\sqrt{5}}{2}\right\}
$$


with $\partial \mathcal{E}_{1}$ having foci at 1 and 0 , and

$$
\mathcal{E}_{2}:=\left\{z \in \mathbb{C}:|z-1|+\left|z-\frac{1+i \sqrt{3}}{2}\right|<\frac{\sqrt{5}}{2}\right\}
$$

with $\partial \mathcal{E}_{2}$ having foci at 1 and $(1+i \sqrt{3}) / 2$.

Subtracting (3.7) from the first equation in (3.6) yields

$$
\frac{b_{1}-\sqrt{3} b_{2}}{\left|w_{1}\right|^{2}}+\frac{1-2 b_{1}}{\left|w_{3}\right|^{2}}=0
$$

where $b_{1}:=\operatorname{Re} b$ and $b_{2}:=\operatorname{Im} b$. Observe that neither of the numerators in (3.8) is zero since neither of the lines $2 b_{1}-1=0, b_{1}-\sqrt{3} b_{2}=0$ intersects $\mathcal{D}$.

By (3.8) we have

$$
\left|w_{1}\right|^{2}=\frac{b_{1}-\sqrt{3} b_{2}}{2 b_{1}-1}\left|w_{3}\right|^{2} .
$$

Plugging this expression into the first identity in (3.6) and simplifying the resulting formulas, we obtain

$$
\left(\frac{b_{1}-\sqrt{3} b_{2}}{2 b_{1}-1}+1\right)\left|w_{3}\right|^{2}+\left(\frac{|b-1|^{2}\left(2 b_{1}-1\right)}{\left(b_{1}-\sqrt{3} b_{2}\right)}+|b|^{2}\right) \frac{1}{\left|w_{3}\right|^{2}}=2 t .
$$

Lemma 2.2 then yields

$$
\left(\frac{b_{1}-\sqrt{3} b_{2}}{2 b_{1}-1}+1\right)\left(\frac{|b-1|^{2}\left(2 b_{1}-1\right)}{\left(b_{1}-\sqrt{3} b_{2}\right)}+|b|^{2}\right)<\frac{5}{4} .
$$

Let us denote the left-hand side of (3.9) by $\phi(b)$. We will now study the behavior of the function $\phi$ in the domain $\mathcal{D}$ and prove:

Lemma 3.2. One has

$$
\phi(b) \geqslant \frac{5}{4}
$$

for all $b \in \mathcal{D}$.

Proof. Let $\mathcal{L}$ be the line $b_{1}+\sqrt{3} b_{2}-1=0$, which we write in parametric form as

$$
\frac{-3+i \sqrt{3}}{8} \sigma+1, \quad \sigma \in \mathbb{R} .
$$

The segment $\mathcal{I}:=\mathcal{L} \cap \mathcal{D}$ is defined by the parameter range

$$
-\frac{1}{\sqrt{15}+3}<\sigma<\frac{1}{\sqrt{15}-3} .
$$

It passes through the common focus of $\partial \mathcal{E}_{1}$ and $\partial \mathcal{E}_{2}$ at 1 (for $\sigma=0$ ) and its closure joins the two points of the intersection $\partial \mathcal{E}_{1} \cap \partial \mathcal{E}_{2}$. By restricting $\phi$ to $\mathcal{I}$, one obtains the quadratic function

$$
\hat{\phi}(\sigma):=\frac{1}{4}\left(3 \sigma^{2}-6 \sigma+8\right),
$$

which is easily seen to be greater than or equal to $5 / 4$ everywhere.

Next, we will restrict $\phi$ to line segments orthogonal to $\mathcal{L}$ and lying in $\mathcal{D}$. Fix $\sigma_{0} \in \mathcal{I}$ and consider the line $\mathcal{L}_{\sigma_{0}}$ given in parametric form as

$$
\frac{1+i \sqrt{3}}{2} \tau+\frac{-3+i \sqrt{3}}{8} \sigma_{0}+1, \quad \tau \in \mathbb{R} .
$$

Clearly, $\mathcal{L}_{\sigma_{0}}$ passes through the point

$$
\mathbf{b}_{0}:=\frac{-3+i \sqrt{3}}{8} \sigma_{0}+1 \in \mathcal{I}
$$


and is orthogonal to $\mathcal{L}$. Restricting $\phi$ to the segment $\mathcal{L}_{\sigma_{0}} \cap \mathcal{D}$, one obtains the function

$$
\hat{\phi}_{\sigma_{0}}(\tau):=\frac{4-3 \sigma_{0}}{4\left(\left(4-3 \sigma_{0}\right)^{2}-16 \tau^{2}\right)}\left(\left(32-48 \sigma_{0}\right) \tau^{2}-9 \sigma_{0}^{3}+30 \sigma_{0}^{2}-48 \sigma_{0}+32\right)
$$

for some range of the parameter $\tau$. Notice that in (3.11) the denominator vanishes exactly at the two points where $\mathcal{L}_{\sigma_{0}}$ intersects the lines $2 b_{1}-1=0, b_{1}-\sqrt{3} b_{2}=0$; these two points lie outside of $\mathcal{D}$.

It is straightforward to see from (3.11) that $\hat{\phi}_{\sigma_{0}}$ takes its minimum at $\tau=0$, i.e., at the point $\mathbf{b}_{0} \in \mathcal{I}$. Indeed, one computes

where

$$
\hat{\phi}_{\sigma_{0}}^{\prime}(\tau)=\frac{32\left(4-3 \sigma_{0}\right) h\left(\sigma_{0}\right)}{\left(\left(4-3 \sigma_{0}\right)^{2}-16 \tau^{2}\right)^{2}} \tau,
$$

$$
h\left(\sigma_{0}\right):=-9 \sigma_{0}^{3}+30 \sigma_{0}^{2}-36 \sigma_{0}+16 .
$$

Let us show that $h\left(\sigma_{0}\right)>0$. On $\overline{\mathcal{I}}$ one has

$$
h^{\prime}(\sigma)=-27 \sigma^{2}+60 \sigma-36<0 .
$$

Therefore, $h\left(\sigma_{0}\right)$ is bounded from below by the value of $h$ at the right endpoint of $\overline{\mathcal{I}}$, i.e., at $1 /(\sqrt{15}-3)($ see $(3.10))$. One easily computes that $h(1)=918 \sqrt{15}-3555>0$, thus $h\left(\sigma_{0}\right)>0$ as required.

Since the function $\hat{\phi}$ is already known to be greater than or equal to $5 / 4$, it follows that $\hat{\phi}_{\sigma_{0}}$ is greater than or equal to $5 / 4$ everywhere.

This completes the proof of the lemma.

As Lemma 3.2 contradicts inequality (3.9), the theorem follows.

\section{A FEW REMARKS}

We conclude the paper by making a number of observations and comments.

Remark 4.1. First of all, it is easy to see that $\tilde{F}_{n}$ is not injective on $M_{t}^{3}$ for every $t \geqslant \sqrt{2}$ and every $n$. Indeed, fix $t \geqslant \sqrt{2}$, let $u \neq 0$ be a real number satisfying

$$
2 u^{2}+\frac{1}{u^{2}}=2 t,
$$

(cf. Lemma 2.2) and consider the following two distinct points in $Q^{3}$ :

$$
w:=\left(u, \frac{1}{u}, u, 0\right), w^{\prime}:=\left(u, 0, u, \frac{1}{u}\right) .
$$

Then $w, w^{\prime} \in M_{t}^{3}$, and it follows from (2.6), (2.15) that $\tilde{F}_{n}(w)=\tilde{F}_{n}\left(w^{\prime}\right)=(u, u, 0)$.

Remark 4.2. The polynomials $R_{n}$ constructed in the proof of Theorem 1.1 do not vanish on the sphere $S^{3} \subset Q^{3}$ (see (2.2)). Therefore, by (2.6), (2.10) and Lemma 2.1 , all the maps $\tilde{F}_{n}$ are nondegenerate at every point of $S^{3}$. It is also clear that all $\tilde{F}_{n}$ are injective on $S^{3}$. Hence, each $\tilde{F}_{n}$ yields an explicit real-analytic totally real embedding of $S^{3}$ to $\mathbb{C}^{3}$. It then follows that each $\tilde{F}_{n}$ is biholomorphic is a neighborhood of $S^{3}$ in $Q^{3}$ and thus defines a CR-embedding of $M_{t}^{3}$ in $\mathbb{C}^{3}$ if $1<t<\tau_{n}$ for some $\tau_{n}$. We did not attempt to determine or estimate $\tau_{n}$ for $n>1$ as the calculations involved appear to be quite hard. Recall that, by Remark 4.1, the map $\tilde{F}_{n}$ fails to be injective on $M_{t}^{3}$ for all $t \geqslant \sqrt{2}$, so we have $\tau_{n} \leqslant \sqrt{2}$.

Remark 4.3. Article [AR] yields a class of maps of the form (2.4), with the restriction of $f$ to $S^{3}$ being a harmonic polynomial given by

$$
\mathcal{P}=\left(\bar{w}_{1} \frac{\partial}{\partial w_{3}}-\bar{w}_{3} \frac{\partial}{\partial w_{1}}\right)\left(\sum_{j=1}^{m} \frac{\mathcal{Q}_{j}}{p_{j}\left(q_{j}+1\right)}\right),
$$


where $\mathcal{Q}_{j}$ is a homogeneous harmonic complex-valued polynomial in $w_{1}, \bar{w}_{1}, w_{3}$, $\bar{w}_{3}$ of total degree $p_{j} \geqslant 1$ in $w_{1}, w_{3}$ and total degree $q_{j}$ in $\bar{w}_{1}, \bar{w}_{3}$, such that the sum $\mathcal{Q}:=\mathcal{Q}_{1}+\cdots+\mathcal{Q}_{m}$ does not vanish on $S^{3}$. Every map of this kind defines a totally real embedding of $S^{3}$ to $\mathbb{C}^{3}$ and therefore a CR-embedding of $M_{t}^{3}$ for $t$ sufficiently close to 1 . One can homogenize $\mathcal{P}$ by multiplying its lower-degree homogeneous components by suitable powers of the polynomial $\left|w_{1}\right|^{2}+\left|w_{3}\right|^{2}$, which is equal to 1 on $S^{3}$. The resulting polynomial $\hat{\mathcal{P}}\left(w_{1}, \bar{w}_{1}, w_{3}, \bar{w}_{3}\right)$ may no longer be harmonic; however, the map

$$
\left(w_{1}, w_{3}\right) \mapsto\left(w_{1}, w_{3}, \hat{\mathcal{P}}\left(w_{1}, \bar{w}_{1}, w_{3}, \bar{w}_{3}\right)\right)
$$

still defines the same totally real embedding of $S^{3}$ to $\mathbb{C}^{3}$ and its extension to $\mathbb{C}^{4}$

$$
\left(w_{1}, w_{2}, w_{3}, w_{4}\right) \mapsto\left(w_{1}, w_{3}, \hat{\mathcal{P}}\left(w_{1}, w_{2}, w_{3}, w_{4}\right)\right)
$$

the same CR-embedding of $M_{t}^{3}$ for $t$ sufficiently close to 1 .

As one example, in $[\mathrm{AR}]$ the authors set

$$
\begin{aligned}
\mathcal{P}=w_{3} \bar{w}_{1} \bar{w}_{3}^{2}-w_{1} \bar{w}_{1}^{2} \bar{w}_{3}+i \bar{w}_{1} \bar{w}_{3}= & \\
& \left(\bar{w}_{1} \frac{\partial}{\partial w_{3}}-\bar{w}_{3} \frac{\partial}{\partial w_{1}}\right)\left(\frac{\left|w_{1}\right|^{4}-4\left|w_{1}\right|^{2}\left|w_{3}\right|^{2}+\left|w_{3}\right|^{4}}{6}+\frac{i\left(\left|w_{3}\right|^{2}-\left|w_{1}\right|^{2}\right)}{2}\right) .
\end{aligned}
$$

Here

$$
\mathcal{Q}=\left|w_{1}\right|^{4}-4\left|w_{1}\right|^{2}\left|w_{3}\right|^{2}+\left|w_{3}\right|^{4}+i\left(\left|w_{3}\right|^{2}-\left|w_{1}\right|^{2}\right),
$$

and it is not hard to see that $\mathcal{Q}$ indeed does not vanish on $S^{3}$. To homogenize $\mathcal{P}$, one multiplies its lowest-degree homogeneous component $i \bar{w}_{1} \bar{w}_{3}$ by $\left|w_{1}\right|^{2}+\left|w_{3}\right|^{2}$, which yields the polynomial

$$
\hat{\mathcal{P}}\left(w_{1}, \bar{w}_{1}, w_{3}, \bar{w}_{3}\right)=(1+i)\left(w_{3} \bar{w}_{1} \bar{w}_{3}^{2}+i w_{1} \bar{w}_{1}^{2} \bar{w}_{3}\right) .
$$

This is the polynomial (up to the factor $1+i$ ) that appears in the main theorem of $[\mathrm{AR}]$. Its natural extension to $\mathbb{C}^{4}$ is

$$
\hat{\mathcal{P}}\left(w_{1}, w_{2}, w_{3}, w_{4}\right)=(1+i)\left(w_{2} w_{3} w_{4}^{2}+i w_{1} w_{2}^{2} w_{4}\right)
$$

In [I1], [I2] we investigated the corresponding map (4.2) for nondegeneracy and injectivity and eventually proved in [I2, Theorem 1.1] that this map yields a CRembedding of $M_{t}^{3}$ to $\mathbb{C}^{3}$ for all $1<t<\sqrt{(2+\sqrt{2}) / 3}$. Most of our effort went into establishing injectivity for $t$ in this range.

The polynomials $P_{n}$ that we utilized in the proof of Theorem 1.1 in Section 2 (see formula (2.15)) are homogeneous by construction, and, except in the case $n=1$, we do not know whether they arise from suitable harmonic polynomials by the homogenization procedure described above. For $n=1$ calculations are easy, and $P_{1}$ is readily seen to come from the inhomogeneous harmonic polynomial

$$
-\frac{1}{6} w_{3} \bar{w}_{1} \bar{w}_{3}^{2}+\frac{1}{6} w_{1} \bar{w}_{1}^{2} \bar{w}_{3}-\frac{i}{2 \sqrt{3}} \bar{w}_{1} \bar{w}_{3} .
$$

Note that for the polynomial $\hat{\mathcal{P}}$ from (4.4), the expression

$$
w_{3} \frac{\partial \hat{\mathcal{P}}}{\partial w_{2}}-w_{1} \frac{\partial \hat{\mathcal{P}}}{\partial w_{4}},
$$

when restricted to $Q^{3}$, has two distinct roots if regarded as a function of the product $w_{3} w_{4}$ (see [I2, formulas $\left.(2.7),(2.8)\right]$ ). For comparison, from $(2.10)$ we see that the analogous expression for $P_{n}$ in place of $\hat{\mathcal{P}}$ is equal to $R_{n}$ whose restriction to $Q^{3}$ has only one (multiple) root (see (2.12)). This makes our polynomials $P_{n}$ easier to deal with in computations. One illustration of this is the proof of Theorem 1.2, where we used $F_{1}$ instead of map (4.2) with $\hat{\mathcal{P}}$ given by (4.4), on which the proof of [I2, 
Teorem 1.1] was based. In particular, formulas (3.1) are less complicated than the corresponding formulas in [I2]. Overall, the proof of Theorem 1.2 is computationally much more transparent than that of [I2, Theorem 1.1].

It is possible that one can investigate the CR-embeddability of $M_{t}^{3}$ in $\mathbb{C}^{3}$ for all $t$ by using other maps of the form (4.2). Note, however, that while it is tempting to take $\mathcal{Q}$ to be a polynomial in $\left|w_{1}\right|^{2},\left|w_{3}\right|^{2}$ (as was done in (4.3)), one should avoid doing so as otherwise map (4.2) will not be injective on $M_{t}^{3}$ with $t \geqslant \sqrt{2}$. This follows exactly as in Remark 4.1; namely (4.2) takes equal values at the two points defined in (4.1).

\section{REFERENCES}

[AR] Ahern, P. and Rudin, W., Totally real embeddings of $S^{3}$ in $\mathbb{C}^{3}$, Proc. Amer. Math. Soc. 94 (1985), 460-462.

[BN] Bell, S. and Narasimhan, R., Proper holomorphic mappings of complex spaces, in Several complex variables, VI, Encyclopaedia Math. Sci., 69, Springer, Berlin, 1990, pp. 1-38.

[BH] Burns, D. and Hind, R., Symplectic geometry and the uniqueness of Grauert tubes, Geom. Funct. Anal. 11 (2001), 1-10.

[F1] Forstnerič, F., Some totally real embeddings of three-manifolds, Manuscripta Math. 55 (1986), 1-7.

[F2] Forstnerič, F., On totally real embeddings into $\mathbb{C}^{n}$, Exposition. Math. 4 (1986), 243-255.

[F3] Forstnerič, F., A totally real three-sphere in $\mathbb{C}^{3}$ bounding a family of analytic disks, Proc. Amer. Math. Soc. 108 (1990), 887-892.

[G1] Gromov, M., Convex integration of differential relations, Math. USSR Izv. 7 (1973), 329343.

[G2] Gromov, M., Partial Differential Relations, Ergebnisse der Mathematik und ihrer Grenzgebiete (3), vol. 9, Springer-Verlag, Berlin, 1986.

[I1] Isaev, A. V., On the classification of homogeneous hypersurfaces in complex space, Internat. J. Math. 24 (2013), no. 8, 1350064 (11 pages, electronic), DOI: 10.1142/S0129167X1350064X.

[I2] Isaev, A. V., On homogeneous hypersurfaces in $\mathbb{C}^{3}$, J. Geom. Anal. 27 (2017), 2044-2054.

[KZ] Kaup, W. and Zaitsev, D., On the CR-structure of compact group orbits associated with bounded symmetric domains, Invent. Math. 53 (2003), 45-104.

[MN] Morimoto, A. and Nagano, T., On pseudo-conformal transformations of hypersurfaces, $J$. Math. Soc. Japan 15 (1963), 289-300.

[SZ] Stout, E. L. and Zame, W. R., A Stein manifold topologically but not holomorphically equivalent to a domain in $\mathbb{C}^{n}, A d v$. Math. 60 (1986), 154-160.

Mathematical Sciences Institute, Australian National University, Acton, Canberra, ACT 2601, Australia

E-mail address: alexander.isaev@anu.edu.au 